\title{
Neutrino Dark Energy With More Than One Neutrino Species
}

\author{
Ole Eggers Bjælde* \\ Department of Physics and Astronomy, University of Aarhus, \\ Ny Munkegade, DK-8000 Aarhus C, Denmark and \\ Center for Cosmology and Particle Physics, Department of Physics, New York University, New York, NY 10003 \\ Steen Hannestad \\ Department of Physics and Astronomy, University of Aarhus, Ny Munkegade, DK-8000 Aarhus C, Denmark
}

(Dated: August 26, 2021)

\begin{abstract}
The mass varying neutrino scenario is a model that successfully explains the origin of dark energy while at the same time solves the coincidence problem. The model is, however, heavily constrained by its stability towards the formation of neutrino bound states when the neutrinos become nonrelativistic. We discuss these constraints and find that natural, adiabatic, stable models with the right amount of dark energy today do not exist. Secondly, we explain why using the lightest neutrino, which is still relativistic, as an explanation for dark energy does not work because of a feedback mechanism from the heavier neutrinos.
\end{abstract}

PACS numbers: 95.36.+x, 98.80.-k

\section{INTRODUCTION}

Precision observations of the cosmic microwave background [1] $\underline{3}$ ], the large scale structure of galaxies [4] , and distant type Ia supernovae [5] [8] have led to a new standard model of cosmology in which the energy density is dominated by dark energy with negative pressure, leading to an accelerated expansion of the universe.

A very interesting proposal is the so-called mass varying neutrino (MaVaN) model [9 11] in which a light scalar field couples to neutrinos, see also [12 51] for background. Because of the coupling, the mass of the scalar field does not have to be as small as the Hubble scale but can be larger, while the model still accomplishes late-time acceleration.

In this paper we discuss the different criteria that need to be fulfilled in order to have a stable, adiabatic mass varying neutrino model with the correct cosmology today - i.e. $\Omega_{\mathrm{DE}}=0.7, \Omega_{\mathrm{M}}=0.3$ and $w \sim-1$. Our aim is to show that it is only possible as long as the scalar field potential resembles a cosmological constant - and hence the model loses its prediction of the current neutrino mass and fails to solve the coincidence problem.

In addition, we discuss the suggestion that the lightest neutrino, which can be relativistic today, may be responsible for dark energy. We find that there is evidence that the relativistic neutrino will feel an instability towards the formation of neutrino nuggets.

In the next section we briefly review the formalism needed to study mass varying neutrinos and in Sec. III we discuss the different criteria in the MaVaN framework. In Sec. IV we discuss MaVaNs with a relativistic neutrino and in Sec. $\mathrm{V}$ we conclude.

\section{MASS VARYING NEUTRINOS}

The idea in the mass varying neutrino scenario 9 -11] is to introduce a coupling between neutrinos and a light scalar field and to identify the coupled fluid with dark energy. In this scenario the neutrino mass $m_{\nu}$ is generated from the vacuum expectation value (VEV) of the scalar field. Thus at scale factor $a$ the pressure $P_{\nu}\left(m_{\nu}(\phi), a\right)$ and energy density $\rho_{\nu}\left(m_{\nu}(\phi), a\right)$ of the uniform neutrino background contribute to the effective potential $V(\phi, a)$ of the scalar field in the following way

$$
V(\phi)=V_{\phi}(\phi)+\left(\rho_{\nu}-3 P_{\nu}\right)
$$

where $V_{\phi}(\phi)$ denotes the fundamental scalar potential.

The energy density and pressure of the scalar field are given by the usual expressions,

$$
\begin{aligned}
\rho_{\phi}(a) & =\frac{1}{2 a^{2}} \dot{\phi}^{2}+V_{\phi}(\phi), \\
P_{\phi}(a) & =\frac{1}{2 a^{2}} \dot{\phi}^{2}-V_{\phi}(\phi) .
\end{aligned}
$$

Defining $w=P_{\mathrm{DE}} / \rho_{\mathrm{DE}}$ to be the equation of state of the coupled dark energy fluid, where $P_{\mathrm{DE}}=P_{\nu}+P_{\phi}$ denotes its pressure and $\rho_{\mathrm{DE}}=\rho_{\nu}+\rho_{\phi}$ its energy density, the requirement of energy conservation gives,

$$
\dot{\rho}_{D E}+3 H \rho_{D E}(1+w)=0 .
$$

Here $H \equiv \frac{\dot{a}}{a}$ and we use dots to refer to the derivative with respect to conformal time. Taking Eq. (3) into account, one arrives at a modified Klein-Gordon equation describing the evolution of $\phi$,

$$
\ddot{\phi}+2 H \dot{\phi}+a^{2} V_{\phi}^{\prime}=-a^{2} \beta\left(\rho_{\nu}-3 P_{\nu}\right)
$$

*Electronic address: oeb@phys.au.dk 
Here and in the following primes denote derivatives with respect to $\phi\left({ }^{\prime}=\partial / \partial \phi\right)$ and $\beta=\frac{d \log m_{\nu}}{d \phi}$ is the coupling between the scalar field and the neutrinos.

In the nonrelativistic case $P_{\nu} \simeq 0$, such that Eq. (1) takes the form

$$
V=\rho_{\nu}+V_{\phi}
$$

Assuming the curvature scale of the potential and thus the mass of the scalar field $m_{\phi}$ to be much larger than the expansion rate of the Universe,

$$
V^{\prime \prime}=\rho_{\nu}\left(\beta^{\prime}+\beta^{2}\right)+V_{\phi}^{\prime \prime} \equiv m_{\phi}^{2} \gg H^{2},
$$

the adiabatic solution to the equation of motion of the scalar field in Eq. (4) applies [11]. As a consequence, the scalar field instantaneously tracks the minimum of its effective potential $V$

$$
V^{\prime}=\rho_{\nu}^{\prime}+V_{\phi}^{\prime}=\beta \rho_{\nu}+V_{\phi}^{\prime}=0
$$

MaVaN models has a possibility of becoming unstable on sub-Hubble scales $m_{\phi}^{-1}<a / k<H^{-1}$ in the nonrelativistic regime of the neutrinos, where the perturbations $\delta \rho_{\nu}$ evolve adiabatically.

In Ref. 27] it is shown that the equation of motion for the neutrino density contrast $[62] \frac{\delta \rho_{\nu}}{\rho_{\nu}}$ in the regime $m_{\phi}^{-1}<a / k<H^{-1}$ can be written as

$$
\begin{array}{r}
\ddot{\delta}_{\nu}+H \dot{\delta}_{\nu}+\left(\frac{\delta P_{\nu}}{\delta \rho_{\nu}} k^{2}-\frac{3}{2} H^{2} \Omega_{\nu} \frac{G_{\text {eff }}}{G}\right) \delta_{\nu} \\
=\frac{3}{2} H^{2}\left[\Omega_{\mathrm{CDM}} \delta_{\mathrm{CDM}}+\Omega_{b} \delta_{b}\right]
\end{array}
$$

where

$$
G_{\text {eff }}=G\left(1+\frac{2 \beta^{2} M_{\mathrm{pl}}^{2}}{1+\frac{a^{2}}{k^{2}}\left\{V_{\phi}^{\prime \prime}+\rho_{\nu} \beta^{\prime}\right\}}\right)
$$

and

$$
\Omega_{i}=\frac{a^{2} \rho_{i}}{3 H^{2} M_{\mathrm{pl}}^{2}} .
$$

Since neutrinos not only interact through gravity, but also through the force mediated by the scalar field, they feel an effective Newton's constant $G_{\text {eff }}$ as defined in Eq. (9). The force depends upon the MaVaN model specific functions $\beta$ and $V_{\phi}$ and takes values between $G$ and $G\left(1+2 \beta^{2} M_{\mathrm{pl}}^{2}\right)$ on very large and small length scales, respectively.

In certain cases of strong coupling neutrinos suffer an instability towards clumping in which case they stop behaving as dark energy [21]. In Ref. [27] a criterion for the stability was developed. $\left(1+\frac{2 \beta^{2} M_{\mathrm{pl}}^{2}}{1+\frac{a^{2}}{k^{2}}\left\{V_{\phi}^{\prime \prime}+\rho_{\nu} \beta^{\prime}\right\}}\right) \Omega_{\nu} \delta_{\nu}<$
$\Omega_{\mathrm{CDM}} \delta_{\mathrm{CDM}}+\Omega_{b} \delta_{b}$. This can be recast in a more convenient form $\frac{2 \beta^{2} M_{\mathrm{pl}}^{2}}{1+\frac{a^{2}}{k^{2}}\left\{V_{\phi}^{\prime \prime}+\rho_{\nu} \beta^{\prime}\right\}} \Omega_{\nu}<\Omega_{\mathrm{M}}$, where we have neglected the effect of baryons compared to cold dark matter and we have assumed the density contrasts of roughly the same order.

\section{MODEL REQUIREMENTS}

In summary we have the following different criteria for an adiabatic MaVaN model.

1) The model needs to satisfy current observations i.e. $w \sim-1$. This is easily fulfilled by demanding $\dot{\phi} \sim 0$ since we know the neutrino contribution to both pressure and energy density is smaller than the scalar field contribution.

2) We want a neutrino mass at present that satisfies the current neutrino mass bounds from cosmology, that is $m_{\nu} \lesssim 1 \mathrm{eV}$. Using this, we get a maximal value for the neutrino density parameter $\Omega_{\nu} \lesssim 0.02$ [3, 4, 52].

3) The model must produce the right cosmology, i.e. $\Omega_{D E} \sim 0.70$. This gives us roughly $\frac{\rho_{\phi}}{\rho_{\nu}} \sim \frac{V_{\phi}}{\rho_{\nu}}>35$.

4) In order to have a prediction for the current neutrino mass, we are looking for an adiabatic model that continuously tracks the minimum of the effective potential, i.e. $V^{\prime}=V_{\phi}^{\prime}+\beta \rho_{\nu}=0$. In addition, to avoid severe fine-tuning, we demand $m_{\phi}^{-1} \ll$ $H^{-1}$ [21, [53]. Hence $V_{\phi}^{\prime \prime}+\left(\beta^{2}+\beta^{\prime}\right) \rho_{\nu} \gg H^{2}$, using a constant coupling gives us $V_{\phi}^{\prime \prime}+\beta^{2} \rho_{\nu} \gg H^{2}$.

5) We want our model to be stable towards the formation of neutrino nuggets at present. This gives the requirement $\frac{2 \beta^{2} M_{\mathrm{pl}}^{2}}{1+\frac{a^{2}}{k^{2}}\left\{V_{\phi}^{\prime \prime}+\rho_{\nu} \beta^{\prime}\right\}} \Omega_{\nu}<\Omega_{\mathrm{M}}$. Since we are in the adiabatic regime, we are looking on scales where $k / a \sim \sqrt{V_{\phi}^{\prime \prime}+\rho_{\nu} \beta^{\prime}}$. Combined with the fact that $\Omega_{\mathrm{M}} \sim 0.30$, this criterion simply reduces to $\frac{2 \beta^{2} M_{\mathrm{pl}}^{2}}{2}=\beta^{2} M_{\mathrm{pl}}^{2}<15$.

Combining the criteria above and using $H^{2}=\frac{\rho_{\text {total }}}{M_{\mathrm{pl}}^{2}} \sim$ $\frac{V_{\phi}}{M_{\mathrm{pl}}^{2}}$, we can see that in the case of a constant coupling we get $\frac{V_{\phi}^{\prime \prime}}{V_{\phi}}+\beta^{2} \frac{\rho_{\nu}}{V_{\phi}} \gg \frac{V_{\phi}}{M_{\mathrm{PL}}^{2} V_{\phi}}$, which reduces to $\frac{V_{\phi}^{\prime \prime} M_{\mathrm{PL}}^{2}}{V_{\phi}}+\frac{15}{35} \gg$ 1 or roughly

$$
\frac{V_{\phi}^{\prime \prime} M_{\mathrm{PL}}^{2}}{V_{\phi}} \gg 1
$$

We propose potentials that resemble those originally proposed in Ref. [1], and in order to be able to explain the coincidence problem we want a potential as simple as 
possible without any type of cosmological constant 63 . However all single field potentials previously studied in this context do not fulfill the constraint Eq. (11).

Hence we need a sort of hybrid model as suggested by [26]. In this type of model we have effectively two minima. One is the true minimum which has zero energy density and the other is the false minimum in which our current universe sits. The offset between the two minima is then what is referred to as the dark energy density. The dynamics of the scalar field is given by the movement of the false minimum with the dilution of the universe. One very nice feature of this model is that it is implemented in supersymmetry and hence explains the stability of a very small scalar field mass. However, since we have an offset it can be interpreted as an effective cosmological constant and as such the hybrid model fails to address the coincidence problem. In addition, to keep the model stable it is suggested that the lightest neutrino is still relativistic and that we associate dark energy with this. We address this issue below.

\section{MAVAN MODEL WITH A RELATIVISTIC NEUTRINO}

We aim at keeping the model as simple as possible, so we propose the existence of just one scalar field with a democratic coupling to all light neutrino species [64]. In that case it is assumed that the lightest neutrino does not clump because of pressure, while the two heavier neutrinos become unstable. In the following we will argue that this is not possible since a feedback from the growth of the heavy neutrino perturbations will cause the relativistic neutrino perturbation to grow as well. The problem in this scenario can be neatly illustrated by the following equation 22]

$$
\delta \rho_{\nu}=\frac{1}{a^{4}} \int q^{2} d q d \Omega \epsilon f_{0}(q) \Psi+\delta \phi \beta\left(\rho_{\nu}-3 P_{\nu}\right) .
$$

The equation explains the growth of the neutrino perturbation and applies to both the interaction between a relativistic neutrino and a scalar field as well as that of a nonrelativistic neutrino and a scalar field.

Let us consider a system consisting of three neutrinos, two heavy nonrelativistic neutrinos $(\mathrm{H})$ and one light relativistic neutrino (L) both interacting with a scalar field. We only have one scalar field so the nonrelativistic neutrinos will inevitably become unstable towards clumping and $\delta \rho_{\nu}(H)$ grows to very large values. For an interaction with a nonrelativistic neutrino, the average scalar field perturbation can be written as [27]

$$
\delta \bar{\phi}=-\frac{\beta \rho_{\nu} \delta_{\nu}}{\left(V_{\phi}^{\prime \prime}+\rho_{\nu} \beta^{\prime}\right)+\frac{k^{2}}{a^{2}}} .
$$

As we can see the scalar field perturbation is effectively proportional to the neutrino perturbation. Note that in reality there will be extra terms in Eq. (13) since we also have a relativistic neutrino, however, these terms will be sub-dominant compared to the terms from the heavier neutrinos. So $\delta \bar{\phi}$ will grow as a result.

Turning our attention to $\delta \rho_{\nu}(L)$, we consider the second term in Eq. (12). $\delta \bar{\phi}$ is growing uninhibited to large values, the coupling $\beta$ is for simplicity assumed of order unity (Planck units) [65]. $\left(\rho_{\nu}-3 P_{\nu}\right)$ is a suppression factor of the order $m / E$ - this factor will act to delay the growth of $\delta \rho_{\nu}(L)$. However, since $\delta \bar{\phi}$ will continue its growth, the inevitable conclusion is that $\delta \rho_{\nu}(L)$ will eventually start to grow. Hence, there exists a type of feedback mechanism between the neutrinos. Note that in this treatment we only considered the interaction between neutrinos of the same type via exchange of the scalar field. If we include couplings between different neutrinos, the feedback mechanism will be even stronger. One could of course argue that we are exactly living in a transition regime when $\delta \rho_{\nu}(L)$ has still not turned unstable. However, that would require serious fine-tuning.

We also investigate a MaVaN model with a relativistic neutrino numerically using the publicly available CMBFAST code [54], where we implement the scenario with two heavy neutrinos with assumed masses $m_{\nu}(H)=$ $0.312 \mathrm{eV}$ and one species of relativistic neutrinos with assumed mass $m_{\nu}(L)=0.0001 \mathrm{eV}$. For the scalar field potential we use a Coleman-Weinberg [55] type scalar field potential similar to the one presented in Refs. [11, 27]

$$
V_{\phi}=V_{0} \log (1+\kappa \phi)
$$

where $\kappa$ and $V_{0}$ are dimensionful constants, which are fixed by demanding the appropriate amount of dark energy in the universe today as well as solving for the minimum of the effective potential. For the mass term of the three neutrinos we choose the same mass term as in [27]

$$
m_{\nu}=\frac{m_{0}}{\phi}
$$

which results in a coupling $\beta=-\frac{1}{\phi}[66]$.

The code calculates the background energy density and pressure of different species from standard integration methods while at the same time it solves for the minimum of the effective potential of the neutrino-scalar field fluid which is

$$
V(\phi)=V_{\phi}(\phi)+\left(\rho_{\nu}(H)-3 P_{\nu}(H)\right)+\left(\rho_{\nu}(L)-3 P_{\nu}(L)\right),
$$

where we remember that there are now two heavy $(\mathrm{H})$ neutrinos and one light (L) neutrino. The expression above can be recast in a more convenient form as [26]

$$
V(\phi)=V_{\phi}(\phi)+m_{\nu}(H) n_{\nu}(H)+\frac{m_{\nu}(L)^{2} T_{\nu}^{2}}{12},
$$


where we introduced the neutrino number density $n_{\nu}$ and neutrino temperature $T_{\nu}$. At the same time as tracking the background we calculate the perturbations to first order such as

$$
\rho_{\nu}=\rho_{\nu}+\delta \rho_{\nu}
$$

This is done for both heavy and light neutrinos using Eq. (12). The results are shown in Fig. 1, where we plot the evolution of the neutrino perturbation and CDM perturbation from a redshift of $z+1=11$ until today.

What happens in Fig. 1 is that the perturbations of the heavy neutrinos, which have already turned nonrelativistic before the range of this plot, are already growing albeit at a slow pace because of the growth-slowing effect of CDM - see Eq. (8). The perturbation of the light neutrino is oscillating (only visible if zoomed in) in this epoch, but also growing slightly as a result of the growth of the coupling $\beta$. The growth-slowing effect of CDM is, however, only temporary and around a reshift of $z+1 \sim 2$ the heavy neutrino perturbation can no longer resist the temptation towards unstable growth. The heavy neutrino perturbation explodes and because of the increased value of $\beta$, the light neutrino perturbation starts exploding as well. We do see that eventually the CDM perturbation also starts exploding and at this moment the linear code is no longer valid. However at this moment both neutrino perturbations are already in the nonlinear regime and will have started forming neutrino bound states. In this regime the MaVaN fluid will no longer act as dark energy and the model breaks down. In conclusion, as a result of a feedback mechanism, the blowing behaviour of the nonrelativistic neutrino density contrast causes the relativistic neutrinos to start clumping as well. Hence the neutrino scalar-field fluid will start acting as a cold dark matter component (clustering neutrinos) and hence cannot be attributed to dark energy.

The reason that the relativistic neutrino is able to clump is that it will acquire an effective mass, thus it cannot be regarded as a relativistic particle. Unfortunately, we cannot use conventional bounds to constrain this effect since the evolution of the neutrino perturbations become nonlinear, i.e. the whole system of equations we are solving, starting with the modified Klein-Gordon equation breaks down. This has the effect that all current bounds are no longer valid, as these are established in the linear regime.

\section{CONCLUSION}

Single scalar field models can be used to explain latetime acceleration in the MaVaN scenario. However, in general using these potentials leads to instabilities towards neutrino bound states unless certain criteria are relaxed. In order to obtain a stable or metastable model we direct the reader to Ref. [27] in which one such model is presented. The crucial thing is that the scalar field

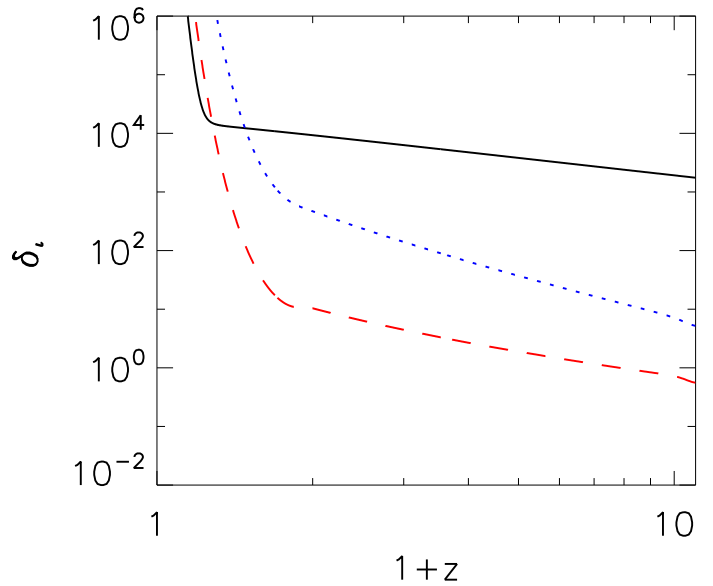

FIG. 1: Density contrasts plotted as a function of redshift for a system consisting of one light and two heavy MaVaN neutrinos each interacting with the same scalar field. The scale is $k=0.1 \mathrm{Mpc}^{-1}$ and we choose the current neutrino masses $m_{\nu}(L)=0.0001 \mathrm{eV}$ and $m_{\nu}(H)=0.312 \mathrm{eV}$ (Note that the choice of current neutrino masses does not affect the result qualitatively). We have chosen $\kappa=1 \times 10^{18}$ in CMBFAST units of $\left(M_{\mathrm{PL}} / \mathrm{Mpc}\right)^{-1 / 2}$ and $V_{0}=1.11 \times 10^{-9}\left(M_{\mathrm{PL}} / \mathrm{Mpc}\right)^{2}$ to fix the cosmology. The solid black line is CDM-density contrast defined as $\frac{\delta \rho_{\mathrm{CDM}}}{\rho_{\mathrm{CDM}}}$, the dotted blue line is the heavy neutrino density contrast $\frac{\delta \rho_{\nu}(H)}{\rho_{\nu}}$, and the dashed red line is the light neutrino density contrast $\frac{\delta \rho_{\nu}(L)}{\rho_{\nu}}$. The heavy neutrinos grow moderately until the coupling becomes large enough for the instabilities to set in. The light neutrino is still relativistic, and its density contrast oscillates as acoustic waves while growing slightly. However, due to a feedback mechanism, the relativistic neutrino density contrast tracks that of the nonrelativistic neutrino especially as the growth of the heavy neutrino perturbation becomes unstable; i.e. both neutrino species will clump. Note that the CDM perturbations also blow up at late times. This is an effect of the system of differential equations breaking down as all parameters go to infinity.

potential resembles that of a cosmological constant. In this case we simply move the fine-tuning problem to that of explaining the small fraction in the power-law - which unfortunately is also rather unnatural.

Accordingly it has been suggested to include an extra scalar field in the treatment. This has some very nice features and is easily capable of obtaining late-time acceleration as well as $\Omega_{\mathrm{DE}}=0.7$ today. However, one drawback is the need for the lightest neutrino to be relativistic today. As was explained above the feedback mechanism will eventually cause the perturbations of the relativistic neutrino to start growing. As a result, the MaVaN fluid will cease to act as dark energy. 


\section{Acknowledgments}

We would like to thank Lily Schrempp, Carsten van de Bruck, Anthony Brookfield, David Mota, and Domenico Tocchini-Valentini for their great collaboration in the paper establishing the underlying framework for this. In addition OEB would like to thank Neal Weiner and Subinoy
Das for some fruitful discussions and NYU for hospitality. We also acknowledge the use of the publicly available CMBFAST code [54].

\section{References}

[1] C. L. Bennett et al., Astrophys. J. Suppl. 148 (2003) 1

[2] D. N. Spergel et al., Astrophys. J. Suppl. 148 (2003) 175

[3] D. N. Spergel et al., arXiv:astro-ph/0603449

[4] M. Tegmark et al., Phys. Rev. D 74, 123507 (2006) arXiv:astro-ph/0608632.

[5] A. G. Riess et al. [Supernova Search Team Collaboration], Astron. J. 116, 1009 (1998)

[6] S. Perlmutter et al. [Supernova Cosmology Project Collaboration], Astrophys. J. 517 (1999) 565

[7] P. Astier et al. [The SNLS Collaboration], Astron. Astrophys. 447, 31 (2006)

[8] W. M. Wood-Vasey et al., arXiv:astro-ph/0701041

[9] P. Q. Hung, arXiv:hep-ph/0010126

[10] P. Gu, X. Wang and X. Zhang, Phys. Rev. D 68, 087301 (2003)

[11] R. Fardon, A. E. Nelson and N. Weiner, JCAP 0410 (2004) 005

[12] R. D. Peccei, Phys. Rev. D 71 (2005) 023527

[13] L. Schrempp, arXiv:astro-ph/0611912

[14] A. Ringwald and L. Schrempp, JCAP 0610, 012 (2006)

[15] D. B. Kaplan, A. E. Nelson and N. Weiner, Phys. Rev. Lett. 93, 091801 (2004)

[16] V. Barger, P. Huber and D. Marfatia, Phys. Rev. Lett. 95, 211802 (2005)

[17] M. Cirelli, M. C. Gonzalez-Garcia and C. Pena-Garay, Nucl. Phys. B 719, 219 (2005)

[18] V. Barger, D. Marfatia and K. Whisnant, Phys. Rev. D 73, 013005 (2006)

[19] P. H. Gu, X. J. Bi, B. Feng, B. L. Young and X. Zhang, arXiv:hep-ph/0512076

[20] H. Li, Z. g. Dai and X. m. Zhang, Phys. Rev. D 71, 113003 (2005)

[21] N. Afshordi, M. Zaldarriaga and K. Kohri, Phys. Rev. D 72 (2005) 065024

[22] A. W. Brookfield, C. van de Bruck, D. F. Mota and D. Tocchini-Valentini, Phys. Rev. Lett. 96 (2006) 061301

[23] A. W. Brookfield, C. van de Bruck, D. F. Mota and D. Tocchini-Valentini, Phys. Rev. D 73 (2006) 083515

[24] R. Takahashi and M. Tanimoto, JHEP 0605 (2006) 021

[25] C. Spitzer, arXiv:astro-ph/0606034.

[26] R. Fardon, A. E. Nelson and N. Weiner, JHEP 0603, 042 (2006) arXiv:hep-ph/0507235.

[27] O. E. Bjaelde, A. W. Brookfield, C. van de Bruck, S. Hannestad, D. F. Mota, L. Schrempp and D. TocchiniValentini, JCAP 0801 (2008) 026 arXiv:0705.2018 [astro-ph]].

[28] M. C. Gonzalez-Garcia and M. Maltoni, Phys. Rept. 460 (2008) 1 arXiv:0704.1800 [hep-ph]].

[29] K. Ichiki and Y. Y. Keum, JCAP 0806 (2008) 005 arXiv:0705.2134 [astro-ph]].

[30] P. H. Gu, H. J. He and U. Sarkar, JCAP 0711 (2007)
016 arXiv:0705.3736 [hep-ph]].

[31] N. Kaloper, Phys. Lett. B $653 \quad$ (2007) 109 arXiv:0706.1977 [astro-ph]].

[32] L. Amendola, M. Baldi and C. Wetterich, arXiv:0706.3064 [astro-ph].

[33] C. Wetterich, Phys. Lett. B $655 \quad$ (2007) 201 arXiv:0706.4427 [hep-ph]].

[34] R. Bean, E. E. Flanagan and M. Trodden, New J. Phys. 10 (2008) 033006 arXiv:0709.1124 [astro-ph]].

[35] R. Bean, E. E. Flanagan and M. Trodden, arXiv:0709.1128 [astro-ph].

[36] R. Takahashi and M. Tanimoto, Phys. Rev. D 77 (2008) 045015 arXiv:0709.2965 [hep-ph]].

[37] P. H. Gu, Phys. Lett. B 661 (2008) 290 arXiv:0710.1044 [hep-ph]].

[38] F. Bauer and L. Schrempp, JCAP 0804 (2008) 006 arXiv:0711.0744 [astro-ph]].

[39] N. Brouzakis, N. Tetradis and C. Wetterich, arXiv:0711.2226 [astro-ph].

[40] J. R. Bhatt, P. H. Gu, U. Sarkar and S. K. Singh, Phys. Lett. B 663 (2008) 83 arXiv:0711.2728 [hep-ph]].

[41] L. Anchordoqui, V. Barger, H. Goldberg and D. Marfatia, Phys. Lett. B 660 (2008) 529 arXiv:0711.4055 [hep$\mathrm{ph}]$.

[42] V. Pettorino and C. Baccigalupi, arXiv:0802.1086 [astro$\mathrm{ph}]$.

[43] D. F. Mota, V. Pettorino, G. Robbers and C. Wetterich, Phys. Lett. B 663 (2008) 160 arXiv:0802.1515 [astro$\mathrm{ph}]$.

[44] A. E. Bernardini and O. Bertolami, Phys. Lett. B 662 (2008) 97 arXiv:0802.4449 [hep-ph]].

[45] L. Schrempp,

[46] P. P. Avelino, L. M. G. Beca and C. J. Martins, Phys. Rev. D 77 (2008) 063515.

[47] K. Ichiki and Y. Y. Keum, arXiv:0803.2274 [hep-ph].

[48] K. Ichiki and Y. Y. Keum, arXiv:0803.3142 [astro-ph].

[49] J. Valiviita, E. Majerotto and R. Maartens, arXiv:0804.0232 [astro-ph].

[50] J. R. Bhatt and U. Sarkar, arXiv:0805.2482 [hep-ph].

[51] B. Li, D. F. Mota and D. J. Shaw, arXiv:0805.3428 [grqc].

[52] A. Goobar, S. Hannestad, E. Mortsell and H. Tu, JCAP 0606, 019 (2006)

[53] M. Kaplinghat and A. Rajaraman, arXiv:astro-ph/0601517

[54] U. Seljak and M. Zaldarriaga, Astrophys. J. 469 (1996) 437.

[55] S. R. Coleman and E. Weinberg, Phys. Rev. D 7, 1888 (1973).

[56] R. Bean and O. Dore, Phys. Rev. D 69, 083503 (2004)

[57] S. Hannestad, Phys. Rev. D 71, 103519 (2005) 
[58] C.-P. Ma and E. Bertschinger, Astrohys. J. 455, 7 (1995).

[59] L. Amendola and D. Tocchini-Valentini Phys. Rev. D 66, 043528 (2002)

[60] T. Koivisto, Phys. Rev. D 72 (2005) 043516

[61] L. Amendola, Phys. Rev. D 69, 103524 (2004)

[62] For more information on the evolution of perturbations see [27, 56 61].

[63] Or scalar field potentials that resemble that of a cosmological constant - e.g. a very small fractional power-law.

[64] One could of course argue that a model with three scalar fields each coupled to one neutrino state would be a nat- ural model to investigate, however, we are merely interested in a proof of concept here, so we stick to the simpler scenario with just one scalar field. In addition this is the standard assumption in the literature.

[65] Note that this assumption makes no difference qualitatively, a smaller coupling would only act to delay the instabilities slightly.

[66] This definition has the advantage that the value of the coupling is determined by the VEV of the field itself, which means that there is no extra free parameter involved. 